\title{
Scalar wormholes in cosmological setting and their instability
}

\author{
Sergey V. Sushkov* \\ Department of General Relativity and Gravitation, Kazan State University, \\ Kremlevskaya str. 18, Kazan 420008, Russia and \\ Department of Mathematics, Tatar State University of Humanities \\ and Education, Tatarstan str. 2, Kazan 420021, Russia \\ Yuan-Zhong Zhang才 \\ Institute of Theoretical Physics, Chinese Academy of Science, P.O. Box 2735 Beijing 100080, China
}

\begin{abstract}
We construct exact nonstatic nonhomogeneous spherically symmetric solutions in the theory of gravity with a scalar field possessing the exponential potential. The solution of particular interest corresponds to the scalar field with negative kinetic energy, i.e. a ghost, and represents two asymptotically homogeneous spatially flat universes connected by a throat. We interpret this solution as a wormhole in cosmological setting. Both the universes and the wormhole throat are simultaneously expanding with acceleration. The character of expansion qualitatively depends on the wormhole's mass $m$. For $m=0$ the expansion goes exponentially, so that the corresponding spacetime configuration represents two de Sitter universes joining by the throat. For $m>0$ the expansion has the power character, so that one has the inflating wormhole connecting two homogeneous spatially flat universes expanding according to the power law into the final singularity.

The stability analysis of the non-static wormholes reveals their instability against linear spherically symmetric perturbations.
\end{abstract}

PACS numbers: 04.20.Jb 98.80.Cq 04.70.Bw

\section{INTRODUCTION}

Wormholes are usually defined as topological handles in spacetime linking widely separated regions of a single universe, or "bridges" joining two different spacetimes [1, 2]. As is well-known [3], they can exist only if their throats contain an exotic matter which possesses a negative pressure and violates the null energy condition. The known classical forms of matter do satisfy the usual energy conditions, hence wormholes should belong to the realm of "unusual" physics. The search of realistic physical models providing the wormhole existence represents an important direction in wormhole physics. Various models of such kind have been considered in the literature, among them scalar fields [4, 5, 6]; wormhole solutions in semiclassical gravity [7]; solutions in Brans-Dicke theory [8]; wormholes on the brane [9]; wormholes supported by matter with an exotic equation of state, namely, phantom energy [10], the generalized Chaplygin gas [1], tachyon matter [12], etc [13, 14].

Recent achievements in observational astrophysics let us to look at wormholes with the new point of view. The observed acceleration of the universe means (at least, in the framework of general relativity) that it is mainly filled by a hypothetical dark energy: the exotic matter with a positive energy density $\rho>0$ and a negative pressure $p=w \rho$ with $w<-1 / 3$. The most exotic form of dark energy is a phantom energy with $w<-1$ [15], for which the null energy condition is violated. It is worth to note that values $w<-1$ not only are not excluded but even are favored by recent observations [16].

The phantom energy, if exists, can be an appropriate material to support wormholes. As a simple model for phantom energy ones consider classical scalar fields with the negative kinetic energy called ghost or phantom scalar fields. ${ }^{1}$ Such the fields can play an important role in cosmology providing the mechanism of the universe acceleration, also they are able to provide the wormhole existence. Yet in 1973 Ellis [4] and, independently, Bronnikov [5] found static spherically symmetric solutions describing wormholes supported by the scalar field with the negative kinetic energy. Subsequent investigations [18, 19] revealed that such solutions are stable against small (linear) perturbations. The stability of static wormholes with ghost fields

\footnotetext{
*Electronic address: sergey.sushkov@ksu.ru

$\dagger$ Electronic address: zyz@itp.ac.cn

1 The words "ghost" and "phantom" are often used on equal footing in papers on gravitation and cosmology. However, it is more correct to distinguish between "phantoms" as "normal" fields possessing an effective equation of state with $w<-1$ and "ghosts" as fields with negative kinetic term. (See a discussion in [17])
} 
seems to be surprising for two reasons, at least. First, different instabilities arise at boundary surfaces dividing ghost and normal field behavior which generally transform these surfaces into singular ones [20]. Second, serious problems with ghost fields appear at the quantum level. Actually, the negative kinetic term leads to the possibility that the energy density may become arbitrarily negative for high frequency oscillations. From the quantum field theory point of view this means the possibility of generating an unlimited amount of particles and antiparticles of usual positive energy matter fields, accompanied by production of equal amount of particles and antiparticles of a negative energy ghost field, i.e. the catastrophic quantum instability of the vacuum 21]. The above arguments let one suppose that the stability of static spherically symmetric wormholes supported by ghost fields is not a general fact.

In this paper we construct and investigate nonstatic spherically symmetric wormholes in the theory of gravity with a ghost scalar field. Such the wormhole represents a throat connecting two universes expanding with an acceleration; the wormhole itself is evolving together with the expanding universes. The stability analysis shows that these solutions turn out to be unstable against linear spherically symmetric perturbations.

The paper is organized as follows. In the section [I] we write down field equations of the theory of gravity with a scalar field and briefly consider general properties of static spherically symmetric wormholes. In the section \II we describe the procedure for generating new non-static solutions being conformally equivalent to "old" static ones. New non-static solutions are analyzed in the section IV] It is shown that the solutions corresponding to the scalar field with negative kinetic energy represent two asymptotically homogeneous spatially flat universes connected by a throat, i.e. wormholes in cosmological setting. In the section $\nabla$ we study a stability of non-static wormholes and show that they turn out to be unstable against linear spherically symmetric perturbations. The section VI summarizes the results obtained. The appendix contains some details of derivation of static spherically symmetric solutions in the theory of gravity with the massless scalar field minimally coupled to the gravitation field.

\section{FIELD EQUATIONS AND STATIC SPHERICALLY SYMMETRIC SOLUTIONS}

Consider the theory of gravity with a real scalar field $\phi$ described by the action

$$
S=\int d^{4} x \sqrt{-g}\left[R-\epsilon g^{\mu \nu} \phi_{, \mu} \phi_{, \nu}-2 V(\phi)\right],
$$

where $g_{\mu \nu}$ is a metric, $g=\operatorname{det}\left(g_{\mu \nu}\right), R$ is the scalar curvature, and $V(\phi)$ is a potential. The value $\epsilon=+1$ corresponds to an ordinary scalar field with positive kinetic energy, and $\epsilon=-1$ to a phantom field, i.e., the scalar field with negative kinetic energy.

Varying the action (1) with respect to $g_{\mu \nu}$ and $\phi$ yields Einstein equations and the equation of motion of the scalar field, respectively:

$$
\begin{aligned}
& R_{\mu \nu}=\epsilon \phi_{, \mu} \phi_{, \nu}+g_{\mu \nu} V(\phi), \\
& \epsilon \nabla^{\alpha} \nabla_{\alpha} \phi=V_{\phi},
\end{aligned}
$$

where $V_{\phi}=d V(\phi) / d \phi$.

The static spherically symmetric solution to the Einstein-scalar equations (2) with $V(\phi) \equiv 0$ was first found by Fisher 22 and then was repeatedly rediscovered and discussed in the literature with various points of view [4, 5, 19, 23, 24, 25, 26, 27]. Below we focus our attention on two general results given by Ellis [4] and Bronnikov [5].

\section{Ellis solution}

In 1973 Ellis [4] exhibited the one-parameter family of general static spherically solutions divided into three qualitatively different classes. His result can be reproduced in the following form: ${ }^{2}$

\footnotetext{
${ }^{2}$ For completeness sake we present details of derivation in the appendix.
} 
Class I. $\lambda>-1 / 2$.

$$
\begin{aligned}
& d s^{2}=-\left(1-\frac{2 m}{\delta r}\right)^{\delta} d t^{2}+\left(1-\frac{2 m}{\delta r}\right)^{-\delta} d r^{2}+\left(1-\frac{2 m}{\delta r}\right)^{1-\delta} r^{2} d \Omega^{2}, \\
& \phi(r)=\delta|\lambda|^{1 / 2} \ln \left(1-\frac{2 m}{\delta r}\right),
\end{aligned}
$$

where $\delta=(1+2 \lambda)^{-1 / 2}$.

Class II. $\lambda=-1 / 2$.

$$
\begin{aligned}
& d s^{2}=-e^{-2 m / r} d t^{2}+e^{2 m / r}\left[d r^{2}+r^{2} d \Omega^{2}\right], \\
& \phi(r)=-\sqrt{2} \frac{m}{r} .
\end{aligned}
$$

Class III. $\lambda<-1 / 2$.

$$
\begin{aligned}
& d s^{2}=-e^{2 m u(r)} d t^{2}+e^{-2 m u(r)}\left[d r^{2}+\left(r^{2}+r_{0}^{2}\right) d \Omega^{2}\right], \\
& \phi(r)=\frac{2 m|\lambda|^{1 / 2}}{r_{0}}\left(\arctan \frac{r}{r_{0}}-\frac{\pi}{2}\right),
\end{aligned}
$$

where $r_{0}=m|1+2 \lambda|^{1 / 2}$, and $u(r)=\frac{1}{r_{0}}\left(\arctan \frac{r}{r_{0}}-\frac{\pi}{2}\right)$.

Above, $\lambda$ and $m$ are two arbitrary parameters of integration, and $d \Omega^{2}=d \theta^{2}+\sin ^{2} \theta d \varphi^{2}$ is the linear element on a unit sphere. It is worth to note that for all three cases the expression for $\phi$ can be represented in the unified form:

$$
\phi(r)=|\lambda|^{1 / 2} \ln \left|g_{t t}\right| .
$$

Note also that $\left|g_{t t}\right|=1-2 m / r+O\left(r^{-2}\right)$ in the limit $r \rightarrow \infty$, hence $m$ plays a role of the asymptotical mass for a distant observer located at $r=\infty$. We will assume that $m \geq 0$.

\section{Bronnikov solution}

In 1973 Bronnikov [5] independently represented another form for the general static spherically symmetric solution of the Einstein-scalar equations (2) with $V(\phi) \equiv 0$. He used the so-called harmonic radial coordinate $\rho$ such that the general static spherically symmetric metric

$$
d s^{2}=-e^{2 \gamma(\rho)} d t^{2}+e^{2 \alpha(\rho)} d \rho^{2}+e^{2 \beta(\rho)} d \Omega^{2}
$$

satisfies the special coordinate condition (the harmonic gauge)

$$
\alpha=2 \beta+\gamma \text {. }
$$

In general, the Bronnikov solution reads

$$
\begin{aligned}
& d s^{2}=-e^{2 m \rho} d t^{2}+\frac{\kappa^{2} e^{-2 m \rho}}{\sinh ^{2}(\kappa \rho)}\left[\frac{\kappa^{2} d \rho^{2}}{\sinh ^{2}(\kappa \rho)}+d \Omega^{2}\right], \\
& \phi(\rho)=\sqrt{2}\left|\kappa^{2}-m^{2}\right|^{1 / 2} \rho .
\end{aligned}
$$

where $\kappa^{2}=m^{2}(1+2 \lambda)$. One may easily check that the relation (9b) for $\phi$ has the form (6). In case $\kappa^{2}=0$ $(\lambda=-1 / 2)$ the metric (9a) reads

$$
d s^{2}=-e^{2 m \rho} d t^{2}+\frac{e^{-2 m \rho}}{\rho^{2}}\left[\frac{d \rho^{2}}{\rho^{2}}+d \Omega^{2}\right]
$$

and in case $\kappa^{2}<0(\lambda<-1 / 2)$,

$$
d s^{2}=-e^{2 m \rho} d t^{2}+\frac{|\kappa|^{2} e^{-2 m \rho}}{\sin ^{2}(|\kappa| \rho)}\left[\frac{|\kappa|^{2} d \rho^{2}}{\sin ^{2}(|\kappa| \rho)}+d \Omega^{2}\right] .
$$

From the relation (9b) one can see that $\phi \sim \rho$, i.e. the scalar field, in fact, plays the role of the harmonic coordinate. Comparing the formula (9b) with (3b), (4b), and (5b) one may find the connection between coordinates $\rho$ and $r$. 


\section{GENERATING NEW NON-STATIC SOLUTION}

Now let us consider the theory (11) with the scalar potential $V(\phi)$ in the Liouville, i.e. exponential form

$$
V(\phi)=V_{0} e^{-k \phi} .
$$

Note that the exponential potential has been considered in numerous papers devoted to cosmological models with scalar fields (see, for instance, 28, 29, 30]). It arises as an effective potential in some supergravity theories or in Kaluza-Klein theories after dimensional reduction to an effective four-dimensional theory [28]. The exponential potential also arises in higher-order gravity theories after a transformation to the Einstein frame [29].

The field equations (2) now yield

$$
\begin{aligned}
& R_{\mu \nu}=\epsilon \phi_{, \mu} \phi_{, \nu}+g_{\mu \nu} V_{0} e^{-k \phi}, \\
& \epsilon \nabla^{\alpha} \nabla_{\alpha} \phi=-k V_{0} e^{-k \phi},
\end{aligned}
$$

In 1995 Fonarev 31] developed the procedure of generating new non-static solutions of the system (13). It was based on the assumption that a non-static solution is conformally equivalent to a static vacuum one. As a result Fonarev constructed the non-static solution being conformally equivalent to the Class I static solution (3). Later, in 32] the new non-static solution being conformally equivalent to the Class III static solution (5) was obtained and analyzed. Now we will present the most general result which can be expressed as the following...

Statement: Let $d \bar{s}^{2}$ and $\bar{\phi}(r)$ be a linear element and a scalar field which form a static spherically symmetric solution of Einstein-scalar equations (13) with $V_{0}=0$, then

$$
\begin{aligned}
& d s^{2}=|\sigma t|^{4 \lambda /(1-2 \lambda)} d \bar{s}^{2}, \\
& \phi(t, r)=\bar{\phi}(r)+\frac{2|\lambda|^{1 / 2}}{1-2 \lambda} \ln |\sigma t|,
\end{aligned}
$$

form a non-static solution of (13) with $V_{0}=2 \sigma^{2} \lambda(6 \lambda-1) /(1-2 \lambda)^{2}$ and $k=|\lambda|^{-1 / 2}$ provided $\lambda \neq 1 / 2$, and

$$
\begin{aligned}
& d s^{2}=e^{2 \sigma t} d \bar{s}^{2}, \\
& \phi(t, r)=\bar{\phi}(r)+\frac{\sigma t}{2},
\end{aligned}
$$

form a non-static solution of (13) with $V_{0}=\sigma^{2} / 2$ and $k=\sqrt{2}$ provided $\lambda=1 / 2$, where $\sigma$ is a free parameter.

Proof. Let $\bar{g}_{\mu \nu}$ and $\bar{\phi}$ be the 'old' static solutions of Einstein-scalar equations (13) with $V_{0}=0$. Consider the conformal transformation of the metric

$$
g_{\mu \nu}=e^{2 \mu(t)} \bar{g}_{\mu \nu},
$$

and suppose that at the same time the scalar field transforms as follows

$$
\phi=\bar{\phi}+\gamma \mu(t),
$$

where $\mu(t)$ is a new indefinite function of $t$, and $\gamma$ is a constant. Using the corresponding transformational properties of the Ricci tensor:

$$
\begin{aligned}
R_{00} & =\bar{R}_{00}-3 \ddot{\mu}, \\
R_{0 i} & =\bar{R}_{0 i}+\dot{\mu} \partial_{i} \ln \left(g_{00}\right), \\
R_{i j} & =\bar{R}_{i j}-\left(\ddot{\mu}+2 \dot{\mu}^{2}\right) g_{i j} g^{00},
\end{aligned}
$$

and taking into account that $\bar{g}_{\mu \nu}$ and $\bar{\phi}$ satisfy the Einstein equations

$$
\bar{R}_{\mu \nu}=\epsilon \bar{\phi}_{, \mu} \bar{\phi}_{, \nu}
$$

it is easy to check that the metric tensor (16) and the scalar field (17) satisfy the field equations (13) provided the function $\mu(t)$ obeys the following two equations:

$$
\begin{aligned}
& \ddot{\mu}+\frac{1-2 \lambda}{2 \lambda} \dot{\mu}^{2}=0, \\
& \dot{\mu}^{2}=-\frac{2 \lambda}{1-6 \lambda} V_{0} e^{(2-1 / \lambda) \mu},
\end{aligned}
$$


and

$$
\gamma=\epsilon|\lambda|^{-1 / 2}, \quad k=|\lambda|^{-1 / 2} .
$$

In case $\lambda \neq 1 / 2$ these equations are compatible and have the solution

$$
\mu(t)=\frac{2 \lambda}{1-2 \lambda} \ln \left|\sigma\left(t-t_{0}\right)\right|
$$

if and only if

$$
V_{0}=\frac{2 \sigma^{2} \lambda(6 \lambda-1)}{(1-2 \lambda)^{2}}
$$

where $\sigma$ and $t_{0}$ are free parameters. Analogously, in case $\lambda=1 / 2$ the solution reads

$$
\mu(t)=2 \sigma\left(t-t_{0}\right)
$$

and

$$
V_{0}=\sigma^{2} / 2 .
$$

Now taking into account Eqs. (16), (17) and making the rescaling $t \rightarrow t+t_{0}$ we arrive at (14) if $\lambda \neq 1 / 2$, and at (15) if $\lambda=1 / 2$.

To complete the proof we consider the scalar field equation (2b). Substituting the expression (17) into (2b) and taking into account that $\bar{\phi}$ satisfies the equation $\bar{\nabla}^{\alpha} \bar{\nabla}_{\alpha} \bar{\phi}=0$ we find

$$
\epsilon \gamma A^{-1} e^{-2 \mu}\left(\ddot{\mu}+2 \dot{\mu}^{2}\right)=k V_{0} e^{-k(\bar{\phi}+\gamma \mu)} .
$$

As is easy to check straightforwardly, this equation is valid for $\bar{\phi}=|\lambda|^{1 / 2} \ln \left|g_{t t}\right|$ and $\mu(t), \gamma, k$, and $V_{0}$ given by the relations $(22+26) .^{3}$

\section{WORMHOLES IN COSMOLOGICAL SETTING}

Now let us analyze the non-static solutions (14a) and (15a) found in the preceding section. First we represent them in the following unified form:

$$
d s^{2}=a^{2}(t)\left[-A d t^{2}+A^{-1} d r^{2}+B r^{2} d \Omega^{2}\right],
$$

where

$$
a(t)=\left\{\begin{array}{cl}
|\sigma t|^{2 \lambda /(1-2 \lambda)}, & \text { if } \lambda \neq 1 / 2, \\
e^{\sigma t}, & \text { if } \lambda=1 / 2,
\end{array}\right.
$$

and functions $A(r)$ and $B(r)$ are defined by the corresponding static solutions (3a), (4a), or (5a), representing the Class I, II, or III, respectively. Note that in the limit $r \rightarrow \infty$ both $A \rightarrow 1$ and $B \rightarrow 1$ for all Classes, and so the metric (28) describes in the asymptotic $r=\infty$ an homogeneous spatially flat universe:

$$
d s^{2}=a^{2}(t)\left[-d t^{2}+d r^{2}+r^{2} d \Omega^{2}\right] .
$$

Using the proper time $T= \pm \int a(t) d t$ gives

$$
d s^{2}=-d T^{2}+b^{2}(T)\left[d r^{2}+r^{2} d \Omega^{2}\right],
$$

with

$$
b(T)=\left\{\begin{array}{cl}
\sigma T, & \text { if } \lambda=1 / 2, \\
\left|\frac{\sigma T}{1-2 \lambda}\right|^{2 \lambda}, & \text { if } \lambda \neq 1 / 2,|\lambda|<\infty \\
e^{\sigma T}, & \text { if }|\lambda|=\infty
\end{array}\right.
$$

\footnotetext{
${ }^{3}$ Notice also that the equation (27) coincides with the $t$-component of Einstein's equations.
} 
The metric (31) explicitly describes an expanding universe with the scale factor $b(T)$. Defining the acceleration parameter $\beta=\ddot{b} b / \dot{b}^{2}$ we find

$$
\beta=\frac{2 \lambda-1}{2 \lambda}
$$

The parameter $\beta$ is negative for $\lambda \in\left(0, \frac{1}{2}\right)$, and so, in this case, the universe is expanding with deceleration, and $\beta$ is positive for $\lambda \in(-\infty, 0) \cup\left(\frac{1}{2}, \infty\right)$, that is, the universe is expanding with acceleration.

Further let us focus on the case of particular interest, i.e. nonstatic wormholes. In this case $\lambda<-1 / 2$, and the solution is given by formulas (14) and (5). Taking into account the relation $r_{0}=m|1+2 \lambda|^{1 / 2}$, we can represent the nonstatic wormhole metric as follows

$$
d s^{2}=|\sigma t|^{-\frac{2\left(m^{2}+r_{0}^{2}\right)}{2 m^{2}+r_{0}^{2}}}\left\{-e^{2 m u(r)} d t^{2}+e^{-2 m u(r)}\left[d r^{2}+\left(r^{2}+r_{0}^{2}\right) d \Omega^{2}\right]\right\},
$$

where $t \in(-\infty, 0), r \in(-\infty, \infty)$, and $u(r)=\frac{1}{r_{0}}\left(\arctan \frac{r}{r_{0}}-\frac{\pi}{2}\right)$. The corresponding solution for the scalar field now reads

$$
\phi(t, r)=\sqrt{2\left(m^{2}+r_{0}^{2}\right)}\left[u(r)+\frac{m \ln |\sigma t|}{2 m^{2}+r_{0}^{2}}\right],
$$

and the potential $V(\phi)=V_{0} e^{-k \phi} \equiv W(t, r)$ takes the following form:

$$
W(t, r)=\frac{\sigma^{2}\left(m^{2}+r_{0}^{2}\right)\left(4 m^{2}+3 r_{0}^{2}\right)}{\left(2 m^{2}+r_{0}^{2}\right)^{2}} \exp \left\{-2 m\left(u(r)+\frac{m \ln |\sigma t|}{2 m^{2}+r_{0}^{2}}\right)\right\} .
$$

The solution (34), (35) depends on three parameters $m, r_{0}$, and $\sigma$. Depending on a value of $m$ it will be convenient to consider separately different cases:

A. $m=0$. In this case the solution (34), (35) takes the especially simple form:

$$
\begin{gathered}
d s^{2}=|\sigma t|^{-2}\left\{-d t^{2}+d r^{2}+\left(r^{2}+r_{0}^{2}\right) d \Omega^{2}\right\}, \\
\phi(r)=\sqrt{2} r_{0} u(r) .
\end{gathered}
$$

Note that in this case the scalar field $\phi$ does not depend on the time coordinate $t$, though the metric (37) is non-static. The potential (36) becomes to be constant:

$$
W(r, T) \equiv 3 \sigma^{2},
$$

and corresponds, in fact, to the positive cosmological constant $\Lambda=3 \sigma^{2}$ in the action (11). Using the proper time $T$ :

$$
-\sigma T=\ln |\sigma t|
$$

yields

$$
d s^{2}=-d T^{2}+e^{2 \sigma T}\left[d r^{2}+\left(r^{2}+r_{0}^{2}\right) d \Omega^{2}\right]
$$

It is easy to see that at each moment of time the metric (41) coincides asymptotically (i.e. in the limit $r \rightarrow$ $\pm \infty$ ) with the de Sitter one, and an intermediate region represents a throat connecting these asymptotically de Sitter regions. Thus, the spacetime (41) is a wormhole joining two de Sitter universes. The instant radius of the throat is equal to the minimal radius of two-dimensional sphere, $r_{t h}=e^{a T} r_{0}$; we see that it grows exponentially with time. Let us calculate now the scalar curvature:

$$
R=12 \sigma^{2}-\frac{2 r_{0}^{2} e^{-2 \sigma T}}{\left(r^{2}+r_{0}^{2}\right)^{2}}
$$

In the limit $r \rightarrow \pm \infty$ as well as in the limit $T \rightarrow \infty$ the scalar curvature has the De-Sitter value $R_{D S}=12 \sigma^{2}$, while at $T=-\infty$ the scalar curvature is singular. This singularity has a clear geometrical interpretation. Namely, at each moment of time the throat is represented as the $2 \mathrm{D}$ sphere of minimal radius. In the limit 
$T \rightarrow-\infty$ the radius of sphere $r_{t h}=e^{\sigma T} r_{0}$ tends to zero, the curvature of sphere goes to infinity, and the corresponding spacetime scalar curvature $R$ becomes to be singular.

It is worth also noting that a metric of the kind of (41) was first introduced a priori by Roman [33], who explored the possibility that inflation might provide a mechanism for the enlargement of submicroscopic, i.e., Planck scale wormholes to macroscopic size.

B. $m>0$. In this case the solution is described by the general formulas (34), (35). Introducing the proper time coordinate by the relation:

$$
|\sigma t|^{-\frac{m^{2}+r_{0}^{2}}{2 m^{2}+r_{0}^{2}}}=|\tilde{\sigma} T|^{-\zeta}
$$

where $\tilde{\sigma}=\left(2+\frac{r_{0}^{2}}{m^{2}}\right) \sigma$ and $\zeta=1+\frac{r_{0}^{2}}{m^{2}}$, we can rewrite the metric (34) in the following form

$$
d s^{2}=-e^{2 m u(r)} d T^{2}+|\tilde{\sigma} T|^{-2 \zeta} e^{-2 m u(r)}\left[d r^{2}+\left(r^{2}+r_{0}^{2}\right) d \Omega^{2}\right],
$$

In two asymptotical regions $r \rightarrow \pm \infty$ the last metric describes homogeneous spatially flat universes: ${ }^{4}$

$$
d s^{2}=-d T^{2}+|\tilde{\sigma} T|^{-2 \zeta}\left[d r^{2}+r^{2} d \Omega^{2}\right],
$$

with the scale factor $a(T)=|\tilde{\sigma} T|^{-\zeta}$ and the scalar curvature

$$
R=\frac{6 \zeta(2 \zeta+1)}{T^{2}} .
$$

The corresponding Hubble parameter $H=\dot{a} / a$ is equal to $\zeta|T|^{-1}$, and the acceleration parameter $\beta=\ddot{a} a / \dot{a}^{2}$ is

$$
\beta=\frac{\zeta+1}{\zeta}=\frac{2 m^{2}+r_{0}^{2}}{m^{2}+r_{0}^{2}}
$$

hence the universes are expanding with an acceleration into a "final" singularity at $T=0_{-}$. The intermediate region $-\infty<r<\infty$ represents a wormhole connecting two universes. The instant radius of the wormhole's throat is equal to the minimal radius of two-dimensional sphere that is achieved at $r=m$ and equal to $r_{t h}=|\tilde{\sigma} T|^{-2 \zeta} e^{-m u(m)}\left(m^{2}+r_{0}^{2}\right)^{1 / 2}$. It is seen that the throat radius grows according to the power law in the course of time.

\section{STABILITY ANALYSIS}

The stability of static wormholes supported by phantom scalar fields was investigated in the literature [18, 19]. It was shown that such the wormholes turn out to be stable against linear spherically symmetric perturbations.

In this section we will study small (linear) spherically symmetric perturbations of the non-static wormhole solution obtained above. For this aim we consider the field perturbation $\phi \rightarrow \phi+\delta \phi$ and the perturbed metric

$$
d s^{2}=|\sigma t|^{-\frac{2\left(m^{2}+r_{0}^{2}\right)}{2 m^{2}+r_{0}^{2}}}\left[-e^{2 u(r)}(1+\delta \gamma) d t^{2}+e^{-2 u(r)}(1+\delta \alpha) d r^{2}+e^{-2 u(r)}(1+\delta \beta)\left(r^{2}+r_{0}^{2}\right) d \Omega^{2}\right],
$$

where the perturbations $\delta \phi, \delta \alpha, \delta \beta$, and $\delta \gamma$ are functions of $t$ and $r$. In perturbation analysis there is the so-called gauge freedom, i.e. that of choosing the frame of reference and the coordinates of the perturbed space-time. Let us choose the following gauge:

$$
\frac{2 m^{2}+r_{0}^{2}}{m^{2}+r_{0}^{2}} e^{4 u(r)} \partial_{r}(-\delta \alpha+2 \delta \beta+\delta \gamma)=\frac{m}{t} \partial_{t}(\delta \alpha+2 \delta \beta-\delta \gamma)+\frac{2 m\left(4 m^{2}+3 r_{0}^{2}\right)}{t^{2}\left(2 m^{2}+r_{0}^{2}\right)} \delta \gamma .
$$

\footnotetext{
${ }^{4}$ In order to obtain Eq. (45) in the region $r \rightarrow-\infty$ one should take into account that $e^{2 m u(r)} \rightarrow 1$ at $r \rightarrow \infty$, and $e^{2 m u(r)} \rightarrow e^{2 \pi m / r_{0}}$ at $r \rightarrow-\infty$, and make an appropriate rescaling.
} 
In this and only in this case the scalar equation (2b) for $\delta \phi$ decouples from the other perturbation equations and reads

$$
e^{4 u(r)}\left[\partial_{r}^{2} \delta \phi+\frac{2 r}{r^{2}+r_{0}^{2}} \partial_{r} \delta \phi\right]=\partial_{t}^{2} \delta \phi-\frac{2\left(m^{2}+r_{0}^{2}\right)}{t\left(2 m^{2}+r_{0}\right)} \partial_{t} \delta \phi-\frac{2 m^{2}\left(4 m^{2}+r_{0}^{2}\right)}{t^{2}\left(2 m^{2}+r_{0}^{2}\right)^{2}} \delta \phi
$$

Separating the variables in Eq. (48): $\delta \phi=\Theta_{\omega}(t) \Phi_{\omega}(r)$, yields

$$
\Phi_{\omega}^{\prime \prime}+\frac{2 r \Phi_{\omega}^{\prime}}{r^{2}+r_{0}^{2}}+\omega^{2} e^{-4 u} \Phi_{\omega}=0
$$

and

$$
\ddot{\Theta}_{\omega}-\frac{2\left(m^{2}+r_{0}^{2}\right)}{t\left(2 m^{2}+r_{0}\right)} \dot{\Theta}_{\omega}+\left[\omega^{2}-\frac{2 m^{2}\left(4 m^{2}+r_{0}^{2}\right)}{t^{2}\left(2 m^{2}+r_{0}^{2}\right)^{2}}\right] \Theta_{\omega}=0
$$

where $\omega^{2}$ is the constant of separation. The equation (49) describes a spatial distribution of perturbations. Its asymptotical solution is

$$
\left.\Phi_{\omega}(r)\right|_{r \rightarrow \infty}=C_{1} \frac{\sin (\omega r)}{r}+C_{2} \frac{\cos (\omega r)}{r}
$$

and

$$
\left.\Phi_{\omega}(r)\right|_{r \rightarrow-\infty}=\tilde{C}_{1} \frac{\sin (\tilde{\omega} r)}{r}+\tilde{C}_{2} \frac{\cos (\tilde{\omega} r)}{r}
$$

where $\tilde{\omega}=e^{2 \pi m / r_{0}} \omega$. In case $\omega^{2}<0$ the perturbations (51) and (52) diverge at $|r|=\infty$, and so this case is unphysical. Therefore, we will analyze the equation (50), which describes an evolution of perturbations, assuming that $\omega^{2} \geq 0$. A general solution of Eq. (50) reads

$$
\Theta_{\omega}(t)=|t|^{\frac{4 m^{2}+3 r_{0}^{2}}{2\left(2 m^{2}+r_{0}^{2}\right)}}\left[D_{1} Y_{\nu}(\omega|t|)+D_{2} J_{\nu}(\omega|t|)\right]
$$

where $J_{\nu}, Y_{\nu}$ are Bessel functions, $D_{1}, D_{2}$ are constants of integrations, and

$$
\nu=\left[\frac{3\left(4 m^{2}+r_{0}^{2}\right)\left(4 m^{2}+3 r_{0}^{2}\right)}{4\left(2 m^{2}+r_{0}^{2}\right)^{2}}\right]^{1 / 2} .
$$

Consider the behavior of $\Theta_{\omega}(t)$ in the limit $t \rightarrow 0 .^{5}$ Taking into account that near zero $J_{\nu}(z) \sim|z|^{\nu}$ and $Y_{\nu}(z) \sim|z|^{-\nu}($ see $[34])$ we find

$$
\left.\Theta_{\omega}(t)\right|_{t \rightarrow 0} \sim D_{1}|\omega t|^{\nu_{-}}+D_{2}|\omega t|^{\nu_{+}}
$$

where

$$
\nu_{ \pm}=\frac{4 m^{2}+3 r_{0}^{2}}{2\left(2 m^{2}+r_{0}^{2}\right)} \pm \nu=\frac{4 m^{2}+3 r_{0}^{2}}{2\left(2 m^{2}+r_{0}^{2}\right)}\left[1 \pm \sqrt{\frac{3\left(4 m^{2}+r_{0}^{2}\right)}{4 m^{2}+3 r_{0}^{2}}}\right]
$$

It is seen that $\nu_{-}$is negative, hence $\Theta_{\omega}(t)$ behaves near zero as $|\omega t|^{\nu_{-}}$. Therefore the ratio $\delta \phi / \phi$, where the non-perturbed solution $\phi$ is given by (35), is diverging at $t=0$. Physically this means that linear scalar field fluctuations are infinitely growing in the course of time. In turn, this means that the considered configuration is unstable against linear spherically symmetric perturbations.

\footnotetext{
${ }^{5}$ Let us remind ourselves that $t \in(-\infty, 0)$, so that the "arrow of time" is directed from $-\infty$ to 0 , and $t=0$ corresponds to the distant future.
} 


\section{CONCLUSIONS}

In this paper we have obtained exact non-static spherically symmetric solutions in the theory of gravity with the scalar field possessing the exponential potential. The solution (34) of particular interest corresponds to the scalar field with negative kinetic energy, i.e. the ghost, and represents two asymptotically homogeneous spatially flat universes connected by a throat. In the other words, one may interpret such the spacetime as a wormhole in cosmological setting. It is important to notice that both the universes and the throat of the wormhole are simultaneously expanding with acceleration. The character of expansion qualitatively depends on the wormhole's mass parameter $m$. In case $m=0$ the expansion goes exponentially, so that the corresponding spacetime configuration, given by the metric (41), represents two de Sitter universes joining by the throat. In case $m>0$ the expansion has the power character, so that the metric (44) describes now the inflating wormhole connecting two homogeneous spatially flat universes expanding according to the power law into the final singularity.

The stability analysis of the non-static wormholes has revealed their instability against linear spherically symmetric perturbations. This result is especially interesting in comparison with the fact that static phantom wormholes are stable in this case (see [18, 19]). Thus, one may suppose that the time dependence makes phantom wormholes to be unstable.

\section{Acknowledgments}

S.S. acknowledge kind hospitality of Institute of Theoretical Physics (Chinese Academy of Science). This project was in part supported by National Basic Research Program of China under Grant No. 2003CB716300 and by NNSFC under Grant No. 90403032. S.S. was also supported in part by the Russian Foundation for Basic Research grants No. 05-02-17344, 05-02-39023.

\section{Appendix}

In the static spherically symmetric case $\phi=\phi(r)$ and the spacetime metric can be taken as

$$
d s^{2}=-A d t^{2}+A^{-1} d r^{2}+B r^{2}\left(d \theta^{2}+\sin ^{2} \theta d \phi^{2}\right),
$$

where $A$ and $B$ are two unknown functions of $r$. The system of equations (2) gets now the following form:

$$
\begin{aligned}
\frac{A^{\prime \prime}}{A}+\frac{A^{\prime} B^{\prime}}{A B}+\frac{2 A^{\prime}}{r A} & =0, \\
-\frac{A^{\prime \prime}}{2 A}-\frac{B^{\prime \prime}}{B}-\frac{2 B^{\prime}}{r B}-\frac{A^{\prime} B^{\prime}}{2 A B}-\frac{A^{\prime}}{r A}+\frac{B^{\prime 2}}{2 B^{2}} & =\epsilon \phi^{\prime 2}, \\
\frac{A^{\prime} B^{\prime}}{A B}+\frac{2 A^{\prime}}{r A}+\frac{B^{\prime \prime}}{B}+\frac{4 B^{\prime}}{r B}-\frac{2}{r^{2} A B}+\frac{2}{r^{2}} & =0,
\end{aligned}
$$

and

$$
\epsilon\left[\phi^{\prime \prime}+\left(\frac{A^{\prime}}{A}+\frac{B^{\prime}}{B}+\frac{2}{r}\right) \phi^{\prime}\right]=0
$$

where a prime means the derivative with respect to $r$. By integrating the equation (58) we find

$$
B=\frac{b_{0}}{r^{2} A^{\prime}},
$$

where $b_{0}$ is a constant of integration. Also, taking into account Eq. (58), we can integrate the field equation (61). As a result we obtain $\phi=\phi_{1}+\phi_{0} \ln A$, where $\phi_{0}$ and $\phi_{1}$ are two constants of integration. Without loss of generality we can put $\phi_{1}=0$, since the action (10) is invariant with respect to the shift $\phi \rightarrow \phi+\phi_{1}$. Now we have

$$
\phi=\phi_{0} \ln A .
$$

The relations (62) and (63) demonstrate to us that $\phi(r)$ and $B(r)$ are expressed via $A(r)$. To obtain an equation for $A$ we substitute Eqs. (62), (63) into (59) and find after some algebra

$$
\left(\frac{A^{\prime \prime}}{A^{\prime}}\right)^{\prime}-\frac{1}{2}\left(\frac{A^{\prime \prime}}{A^{\prime}}\right)^{2}=\lambda\left(\frac{A^{\prime}}{A}\right)^{2},
$$


where $\lambda \equiv \epsilon \phi_{0}^{2}$ is a new parameter with values lying in the interval $(-\infty, \infty)$ depending on $\epsilon$ and $\phi_{0}$. Note that $\phi_{0}=|\lambda|^{1 / 2}$, and hence $\phi=|\lambda|^{1 / 2} \ln A$. A general solution of Eq. (64) reads

$$
A(r)=\exp \left\{\frac{2}{\sqrt{1+2 \lambda}} \operatorname{arctanh}\left[\frac{C_{1}\left(r+C_{2}\right)}{2 \sqrt{1+2 \lambda}}\right]+C_{3}\right\},
$$

where $C_{1}, C_{2}$, and $C_{3}$ are constants of integration. To analyze the obtained solution it will be more convenient to separate three cases depending on values of $\lambda$. Namely,

Case I. $\lambda>-1 / 2$. In this case the solution (65) reduces to

$$
A(r)=A_{1}\left(1-\frac{2 m}{\delta\left(r-r_{1}\right)}\right)^{\delta}
$$

where $\delta=(1+2 \lambda)^{-1 / 2}$, and $A_{1}, m$, and $r_{1}$ are arbitrary constants. By making an appropriate rescaling of coordinates $t$ and $r$ we can put $A_{1}=1$ and $r_{1}=0$, so that $A=(1-2 m / \delta r)^{\delta}$. Now from Eq. (62) we obtain $B=\left(b_{0} / 2 m\right)(1-2 m / \delta r)^{1-\delta}$. The value of $b_{0}$ is not free. To fix it we should substitute $A(r)$ and $B(r)$ into Eq. (60). This yields $b_{0}=2 \mathrm{~m}$. Remembering that $\phi=|\lambda|^{1 / 2} \ln A$ we come, finally, to the following result:

$$
\begin{aligned}
& d s^{2}=-\left(1-\frac{2 m}{\delta r}\right)^{\delta} d t^{2}+\left(1-\frac{2 m}{\delta r}\right)^{-\delta} d r^{2}+\left(1-\frac{2 m}{\delta r}\right)^{1-\delta} r^{2}\left(d \theta^{2}+\sin ^{2} \theta d \varphi^{2}\right), \\
& \phi(r)=\frac{|\lambda|^{1 / 2}}{(1+2 \lambda)^{1 / 2}} \ln \left(1-\frac{2 m}{\delta r}\right)
\end{aligned}
$$

where $\delta=(1+2 \lambda)^{-1 / 2}$. Note that the solution in the form (66) has been given by Buchdahl [25].

Case II. $\lambda=-1 / 2$. In this case the solution (65) reads

$$
A(r)=A_{1} e^{-2 m /\left(r-r_{1}\right)}
$$

where $A_{1}, m$, and $r_{1}$ are arbitrary constants. An appropriate rescaling coordinates $t$ and $r$ yields $A=e^{-2 m / r}$. The functions $B(r)$ and $\phi(r)$ are found in the same way as above. Finally, we obtain

$$
\begin{aligned}
& d s^{2}=-e^{-2 m / r} d t^{2}+e^{2 m / r}\left[d r^{2}+r^{2}\left(d \theta^{2}+\sin ^{2} \theta d \varphi^{2}\right)\right] \\
& \phi(r)=-\sqrt{2} \frac{m}{r} .
\end{aligned}
$$

First this solution has been exhibited by Yilmaz [24].

Case III. $\lambda<-1 / 2$. In this case the solution (65) takes the following form:

$$
A(r)=A_{1} \exp \left\{\frac{2}{|1+2 \lambda|^{1 / 2}} \arctan \left(\frac{r-r_{1}}{r_{0}}\right)\right\},
$$

where $A_{1}, r_{0}$, and $r_{1}$ are arbitrary constants. Analogously to the previous cases, appropriate rescaling coordinates $t$ and $r$ yields $A_{1}=\exp \left(-m \pi / 2 r_{0}\right)$ and $r_{1}=0$, so that $A(r)=e^{2 m u(r)}$, where we have denoted $u(r)=\left(1 / r_{0}\right)\left[\arctan \left(r / r_{0}\right)-\pi / 2\right]$, and $m / r_{0}=|1+2 \lambda|^{-1 / 2}$. Now we find $B(r)$ and $\phi(r)$ and come to the solution

$$
\begin{aligned}
& d s^{2}=-e^{2 m u(r)} d t^{2}+e^{-2 m u(r)}\left[d r^{2}+\left(r^{2}+r_{0}^{2}\right)\left(d \theta^{2}+\sin ^{2} \theta d \varphi^{2}\right)\right], \\
& \phi(r)=\frac{2|\lambda|^{1 / 2}}{|1+2 \lambda|^{1 / 2}}\left(\arctan \frac{r}{r_{0}}-\frac{\pi}{2}\right) .
\end{aligned}
$$

[1] M. S. Morris and K. S. Thorne, American Journal of Physics 56, 395 (1988).

[2] M. Visser, Lorentzian Wormholes: from Einstein to Hawking, (American Institute of Physics, Woodbury, 1995).

[3] D. Hochberg and M. Visser, Phys. Rev. D 56, 4745 (1997);

D. Hochberg and M. Visser, Phys. Rev. D 58, 044021 (1998).

[4] H. Ellis, J. Math. Phys. 14, 104 (1973).

[5] K. A. Bronnikov, Acta Phys. Polonica B 4, 251 (1973). 
[6] T. Kodama, Phys. Rev. D 18, 3529 (1978); C. Barceló and M. Visser, Phys. Lett. B 466, 127 (1999); C. Barceló and M. Visser, Class. Quantum Grav. 17, 3843 (2000); S. V. Sushkov, S.-W. Kim, Class. Quantum Grav. 63, 4909 (2002).

[7] S. V. Sushkov, Phys. Lett. A164, 33 (1992); D. Hochberg, A. Popov, S. V. Sushkov, Phys. Rev. Lett. 78, 2050 (1997); S. V. Krasnikov, Phys. Rev. D 62, 084028 (2000).

[8] K. K. Nandi, B. Bhattacharjee, S. M. K. Alam, and J. Evans, Phys. Rev. D 57, 823 (1998).

[9] L. A. Anchordoqui and S. E. Perez Bergliaffa, Phys. Rev. D 62, 067502 (2000); K. A. Bronnikov and S.-W. Kim, Phys. Rev. D 67, 064027 (2003); M. La Camera, Phys. Lett. B573, 27 (2003); F. S. N. Lobo, arXiv:gr-qc/0701133

[10] S. V. Sushkov, Phys. Rev. D 71, 043520 (2005); F. S. N. Lobo, Phys. Rev. D 71, 084011 (2005).

[11] F. S. N. Lobo, Phys. Rev. D 73, 064028 (2006).

[12] A. Das and Sayan Kar, Class. Quantum Grav. 22, 3045 (2005).

[13] Today the list of references concernig various aspects of wormhole physics numbers hundreds of items. To find more references dated till 1995 the reader can see the book by Visser [2]. A more complete list of up-to-date publications as well as an introduction into a modern state of affairs in wormhole physics and related fields can be found in an excellent review by Lobo [14].

[14] F. S. N. Lobo, arXiv:0710.4474.

[15] R. Caldwell, Phys. Lett. B 545, 23 (2002).

[16] Tonry J.L. et al.,Astrophys. J. 594, 1 (2003); Alam U., Sahni V., Saini T.D., Starobinsky A.A., Mon. Not. Roy. Astron. Soc. 354, 275 (2004); Choudhury T.R., Padmanabhan T., Astron. Astrophys. 429, 807 (2005); Alcaniz J. S., Phys. Rev. D 69, 083521 (2004).

[17] K. A. Bronnikov, A. A. Starobinsky, JETP Lett. 85, 1 (2007).

[18] K.A. Bronnikov, G. Clement, C.P. Constantinidis, J.C. Fabris, Phys. Lett. A243, 121 (1998); K. A. Bronnikov, G. Clement, C.P. Constantinidis, J.C. Fabris, Grav.Cosmol. 4, 128 (1998).

[19] C. Armendáriz-Picón, Phys. Rev. D 65, 104010 (2002).

[20] K. A. Bronnikov and S. V. Grinyok, Grav. \& Cosmol. 10, 237 (2004); A. Vikman, Phys. Rev. D 71, 023515 (2005); F. Cannata and A.Yu. Kamenshchik, gr-qc/0603129 (2006).

[21] J. M. Cline, S. Jeon and G. D. Moore, Phys. Rev. D 70, 043543 (2004).

[22] I. Z. Fisher, Zh. Eksp. Teor. Fiz. 18, 636 (1948) [in Russian]; arXiv:gr-qc/9911008

[23] O. Bergmann and R. Leipnik, Phys. Rev. 107, 1157 (1957);

[24] H. Yilmaz, Phys. Rev. 111, 1417 (1958);

[25] H. A. Buchdahl, Phys. Rev. 115, 1325 (1959).

[26] A. I. Janis, D. C. Robinson, J. Winicour, Phys. Rev. 186, 1729 (1969).

[27] M. Wyman, Phys. Rev. D 24, 839 (1981).

[28] J. J. Halliwell, Phys. Lett. 185B, 341 (1987).

[29] J. D. Barrow, Nucl. Phys. B 296, 697 (1988); J. D. Barrow, S. Cotsakis, Phys. Lett. 214B, 515 (1988); S. Cotsakis, P. J. Saich, Class. Quantum Grav. 11, 383 (1994); A. B. Burd, J. D. Barrow, Nucl. Phys. B 308, 929 (1988).

[30] F. Lucchin, S. Matarrese, Phys. Rev. D 32, 1316 (1985); A. Feinstein, J. Ibáñez, Class. Quantum Grav. 10, L227; 93 (1993); J. M. Aguirregabiria, A. Feinstein, J. Ibáñez, Phys. Rev. D 48, 4662; Phys. Rev. D 484669 (1993); A. R. Liddle, A. Mazumdar, F. E. Schunck, Phys. Rev. D 58, 061301(R) (1998); K. A. Malik, D. Wands, Phys. Rev. D 59, 123501 (1999); E. J. Copeland, A. Mazumdar, N. J. Nunes, Phys. Rev. D 60, 083506 (1999); T. Barreiro, E. J. Copeland, N.J. Nunes, Phys. Rev. D 61, 127301 (2000).

[31] O. A. Fonarev, Class. Quantum Grav. 12, 1739 (1995).

[32] S. V. Sushkov, S.-W. Kim, Gen. Rel. Grav. 36, 1671 (2004).

[33] T. A. Roman, Phys. Rev. D 47, 1370 (1993).

[34] M. Abramowitz and I.A. Stegun, "Handbook of Mathematical Functions", US National Bureau of Standards, Washington, 1964. 